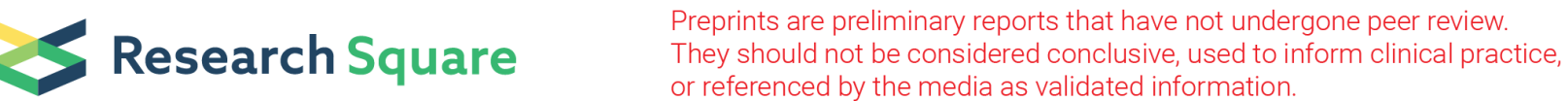

\section{D printed guiding device assisted bilateral crossing cervical microendoscopic laminoplasty for cervical spondylotic myelopathy: a study protocol for a randomized controlled trial}

\section{Chunlin Zhang ( $D$ zzclin@126.com )}

Zhengzhou University First Affiliated Hospital https://orcid.org/0000-0001-9565-4485

\section{Yang Feng}

Zhengzhou University First Affiliated Hospital

\section{Xu Yan}

Zhengzhou University First Affiliated Hospital

\section{Tengyue Yang}

Zhengzhou University First Affiliated Hospital

\section{Chenglong Shao}

Zhengzhou University First Affiliated Hospital

\section{Lijie Shang}

Zhengzhou University First Affiliated Hospital

\section{Yang Liu}

Zhengzhou University First Affiliated Hospital

\section{Yanyu Wu}

Zhengzhou University First Affiliated Hospital

\section{Xiao Zhao}

Zhengzhou University First Affiliated Hospital

\section{Study protocol}

Keywords: Cervical spondylotic myelopathy, 3D Printing, Guiding device, Bilateral crossing cervical microendoscopic laminoplasty, Randomized controlled trial

Posted Date: July 31st, 2019

DOI: https://doi.org/10.21203/rs.2.12206/v1

License: (c) (i) This work is licensed under a Creative Commons Attribution 4.0 International License. Read Full License 


\section{Abstract}

Background Cervical microendoscopic laminoplasty (CMEL) is an important surgical method for the treatment of cervical spondylotic myelopathy (CSM). It has the advantages of minimal incision and quick recovery, meanwhile, it also has disadvantages, such as its tedious to install the implants and long operative time. This study proposes a new method of 3D printed guiding device assisted bilateral crossing cervical microendoscopic laminoplasty for CSM, which can further improve the accuracy and efficiency of surgery and shorten the operative time. Methods/design Patients who met the surgical criteria will be randomly divided into experimental group and control group. The experimental group will be treated with 3D printed guiding device assisted bilateral crossing cervical microendoscopic laminoplasty. The control group was treated with CMEL. The primary outcomes will be incision length, operative time, intraoperative blood loss and adverse events. The secondary outcomes will be the Japanese Orthopedic Association (JOA) score, the visual analogue scales (VAS) for neck and shoulder pain, cervical curvature index $(\mathrm{CCl})$, range of motion (ROM), the sagittal diameter of the spinal canal, and the healing of the grooves. These parameters will be evaluated preoperatively, intraoperatively, 1 week postoperatively, as well as 3, 6, 12, 24 months postoperatively. Discussion This study was the first randomized controlled trial to compare 3D printed guiding device assisted bilateral crossing cervical microendoscopic laminoplasty with CMEL. The results of this study may facilitate the development of surgical treatment of cervical spondylotic myelopathy.

\section{Background}

Surgical treatment of CSM has been developed for decades [1]. Cervical laminoplasty is an important method for the treatment of CSM. The methods include open-door laminoplasty and bilateral open-door laminoplasty. Although laminoplasty had a satisfactory long-term effect, postoperative neck and shoulder pain, decrease in lordosis and ROM is observed in many patients [2-4].

In recent years, some minimally invasive surgical procedures for CSM had been reported [5-13], such as microendoscopic decompression, minimally invasive cervical microendoscopic laminectomy. In 2007, we developed a new minimally invasive procedure for multi-level CSM, namely cervical microendoscopic laminoplasty (CMEL) [5], the key points of CMEL are bilateral long groove-shaped decompression, the spinous process-ligament complex retroposition in the sagitta plane, and the fixation of titanium miniplate. This surgical procedure represents a future development direction, which can significantly reduce trauma, meanwhile postoperative follow-up show that cervical disc may spontaneously be absorbed. However, there are still some difficulties in installing titanium mini-plate under the endoscope and achieving the retroposition of the spinous process-ligament complex: (a) Poor control accuracy: the retroposition of the spinous process-ligament complex relied on intraoperative manual lifting, which was inconvenient to precisely control; (b) Difficulty in operation: because the titanium mini-plate must be matched with the surface of lamina, the sliding grooves of the bilateral mini-plate cannot be parallel to the sagittal plane of the human body, which led to some troubles in the retroposition of the spinous process-ligament complex in this way; (c) The operation was cumbersome: because the mini-plate had a 
certain length and was installed on the same side of the operation, which led to a larger incision. The structure of the spinous process was big on the top and small on the bottom, which led to twist screws on the spinous process side was difficult, and the operation was laborious and time-consuming; (d) Endoscopic operation was prone to skew: due to the anatomical markers cannot be used for positioning under direct vision, the long groove-shaped decompression was prone to skew, which may miscut the bone of the screw installation site, resulting in the difficulty in the installation of titanium mini-plate. These factors had seriously affected the promotion of this minimally invasive technology. Therefore, improving surgical precision, reducing surgical trauma, reducing the difficulty of the operation, and improving the efficiency of surgery have become important problems to be solved urgently.

To solve the above operational problems, we have developed a new surgical operating device for the bilateral crossing cervical microendoscopic laminoplasty (the patent application number is CN201910086277). Based on surface features of the lamina, a 3D printed guiding device is used for locating and navigating.

For all we know, there are no randomized controlled trials of 3D printed guiding device assisted bilateral crossing cervical microendoscopic laminoplasty versus CMEL for CSM. In this trial, we will conduct a randomized controlled trial (RCT) to compare 3D printed guiding device assisted bilateral crossing cervical microendoscopic laminoplasty with CMEL to demonstrate this new surgical method is safer, easier and more effective.

\section{Methods/design}

\section{Study design}

The study is a single-centre, prospective, randomized controlled trial, the objective of which is to evaluate the efficiency and safety of 3D printed guiding device assisted bilateral crossing cervical microendoscopic laminoplasty for CSM. A total of 256 patients will be randomly divided into experimental group and control group in a 1:1 ratio. The experimental group will be treated with 3D printed guiding device assisted bilateral crossing cervical microendoscopic laminoplasty. The control group was treated with CMEL. The flow chart of the study process is as follows in Fig. 1.

This trial is reported in accordance with the Standard Protocol Items: Recommendations for Intervention Trials (SPIRIT) guidelines [14] (Fig. 2, Additional file 1)

\section{Ethical approval}

The trial meets the requirements of the Helsinki Declaration formulated by the World Medical Association, and it has been reviewed and approved by the institutional ethics review board of the First Affiliated Hospital of Zhengzhou University (Approved number: KY-2019-LW-049). All participants will be required to sign an informed consent. The protocol had been registered in the Chinese Clinical Trial Registry 
(ChiCTR). In 2007, ChiCTR was appointed to be the representative registry of China and joined the WHO ICTRP. The protocol number is ChiCTR1900023100.

\section{Participants}

The study is a RCT conducted at the First Affiliated Hospital of Zhengzhou University. Baseline characteristics of patients, such as age, gender, time of onset, symptoms of neck and limbs will be provided. Treatment decisions are made based on baseline characteristics. Surgical treatment is the best choice for patients with ineffective conservative treatment, aggravated pain, and even muscle paralysis.

Inclusion criteria

1. Age $>16$ years old;

2. 2 to 5 levels of cervical degenerative disc disease or spinal canal stenosis at C3-C7;

3. Diagnosis of degenerative disc disease or spinal canal stenosis verified by medical history and physical examination;

4. MRI and CT showed that the canal/vertebral body sagittal ratio was less than 0.75 , but no focal protrusion, fragment protrusion or nerve root compression;

5. Strict conservative treatment was ineffective for more than 3 months.

Exclusion criteria

1. Single-level spinal canal stenosis or degenerative disc disease;

2. Cervical spondylotic radiculopathy, radiological evidence of nerve root compression caused by disc herniation;

3. Severe osteoporosis;

4. Greater than grade 1 spondylolisthesis;

5. Unwilling to sign informed consent for the study.

\section{Recruitment}

The trial recruited the inpatients of the Department of Orthopaedics, the First Affiliated Hospital of Zhengzhou University, and distributed the research plan leaflets to the patients. After reading the leaflets, the patients and their families voluntarily decided whether to participate in the trial. Screening was conducted according to inclusion and exclusion criteria, patients could only be included in the study after signing the informed consent.

\section{Randomisation and blinding}

Patients will be randomly divided into an experimental group and a control group in a 1:1 ratio. It is impossible to blind the participants and surgeons, only the result evaluators are blinding, and these 
evaluators are not involved in patient management. To prevent bias, the patient's randomization process will be performed by a third party.

\section{Sample size calculation}

We carried out a power analysis to evaluate the required sample size to show safety with a power (1- $\beta$ ) of 0.8 and $a$ of 0.05 . According to the relevant literature [5], the excellent and good rate of the control group was $84.4 \%$. We hypothesise that the excellent and good rate of the experimental group could be $95 \%$. We carried out a two independent samples t-tests using PASS (Power Analysis and Sample Size), and obtained a result of 128 .

\section{Surgical procedures}

\section{Experimental group}

Before the operation, the patient underwent a CT scan of the cervical spine by 64 slice spiral CT (Siemens, Germany). After the scan, the original data was exported and stored in the standard STL format, and the STL format file was imported into E3D Medical Software for 3D reconstruction. The technique of 3D reconstruction which extracts the posterior surface features of the cervical spine, may help perform preoperative simulation on the obtained 3D model and determine some important information, such as the position of the decompression grooves, the entry point and depth of the lateral mass screws, the horizontal distance between the decompression groove and the entry point of the lateral mass screw on the same side, also the entry point and angle of the connecting rods pass through the root of the spinous process. A guiding device includes two guiding tubes, two guiding grooves and two aiming devices. The guiding tubes, the guiding grooves and the aiming devices were designed by using E3D Medical Software (Fig. 3), then printed by 3D printing technology, ensuring that the customized ends of the bilateral guiding tubes were perfectly matched with the bilateral lamina surface. Four indicators can be installed on the outside of the guiding tube. When customized ends of the bilateral guiding tubes were perfectly matched with the surface of lamina, the protruding height of the four indicators was identical.

After initiation of general anesthesia, the patient was placed in a prone position, the head was fixed. Under fluoroscopic guidance, the targeting level was confirmed. Two lateral incisions approximately $20 \mathrm{~mm}$ in length were made at the targeting level. Then, the dilator was inserted into the bilateral incision in order, until it contacted the lamina. The dilator was removed after the guiding tube was inserted. The two guiding tubes were connected with the bilateral guiding grooves. The customized ends of the bilateral guiding tubes were perfectly matched with the surface of lamina by adjusting the guiding tubes, and then confirmed by the indicators(Fig. 4A). The guiding tube on the one side was removed, then the endoscope was inserted into the guiding tube on the contralateral side. Endoscopic partial laminotomy was initiated using a high-speed drill to roughen the conjunction of the lamina and the lateral mass, and the ventral cortex of the lamina was completely removed by using a $2 \mathrm{~mm}$ Kerrison laminectomy rongeur. Then a groove of approximately 2 to $3 \mathrm{~mm}$ wide was made at the junction (Fig. 4B). The guiding tube was removed towards the lateral side by a certain distance(this distance is the horizontal distance between 
the decompression groove and the entry point of the lateral mass screw on the same side). Meanwhile, with the help of the working sleeve, the Magerl technique was used to fix the lateral mass screw at the lateral mass (Fig. 4C) [15]. The guiding groove was removed, the aiming device was installed on the guiding tube in the next, then the connecting rod passed through the root of the spinous process by using the aiming device and was fixed at the lateral mass screw in the last (Fig. 4D). Thereafter, the working sleeve was removed, the two guiding tubes were reconnected with the bilateral guiding grooves, and the same process was repeated on the contralateral side. Then two working sleeves were connected with the bilateral lateral mass screws. By adjusting the retroposition locking mechanism of the lateral mass screw to make the bilateral connecting rods retroposition 1 to $3 \mathrm{~mm}$ on the sagittal plane, therefore, the spinous process-ligament complex and the deep extensor muscle group also retroposition 1 to $3 \mathrm{~mm}$ on the sagittal plane (Fig. 4E,F).

After all of the above steps had been completed, the surgical cavity was flushed, the bleeding was tightly stopped, and a drainage tube was placed. Finally, the fascia layer and skin incision were closed by using standard techniques.

Control group

After initiation of general anesthesia, the patient was placed in a prone position, the head was fixed. Under fluoroscopic guidance, the targeting level was confirmed. Then, a midline skin incision approximately $20 \mathrm{~mm}$ in length was made at the targeting level. Bilateral fascial layers were incised and a hole was drilled in each spinous process. Silk suture was used to thread through the hole, and prepared for suspension of the posterior column structure. Then, the dilator was inserted into the left/right side of the spinous process in order, until it contacted the lamina. The dilator was removed after the working sleeve of endoscope was placed on the surface of the lamina through it. Endoscopic partial laminotomy was initiated using a high-speed drill to roughen the conjunction of the lamina and the lateral mass, and the ventral cortex of the lamina was completely removed by using a $2 \mathrm{~mm}$ Kerrison laminectomy rongeur. Thereafter, a groove of approximately 2 to $3 \mathrm{~mm}$ wide was made at the conjunction. The working sleeve of endoscope could be swung cranially and caudally, and the small incision was long enough for three continuous levels simultaneously. The silk suture was pulled dorsally, then under its protection, the same process was repeated on the contralateral lamina. After decompression of the contralateral lamina was completed, the spinous process-ligament complex and the deep extensor muscle group retroposition 1 to $3 \mathrm{~mm}$ on the sagittal plane by pulling the silk suture. Thereafter, the medial side of the mini-plate was fixed at the root of the spinous process with a small screw, the lateral side of the mini-plate fixed at the lateral mass using the Magerl technique.

After all of the above steps had been completed, the surgical cavity was flushed, the bleeding was tightly stopped, and a drainage tube was placed. Finally, the fascia layer and skin incision were closed by using standard techniques.

\section{Outcome measure}


Primary outcomes

Incision length, operative time, intraoperative blood loss and adverse events.

Secondary outcomes

1. JOA

2. VAS

3. $\mathrm{CCl}$

4. ROM

5. The sagittal diameter of the spinal canal.

6. The healing of the grooves.

The data will be collected preoperatively; intraoperatively; on 1 week postoperatively; as well as at $3,6,12$, 24 months postoperatively.

\section{Adverse event}

Researchers should record the adverse events that occurred after cervical spine surgery and report them to the First Affiliated Hospital of Zhengzhou University for a time limit of 24 hours. Specific adverse events include neck and shoulder pain, incision infection, spinal cord injury, nerve root injury, the dural sac tear, major vascular injury, screws shedding and loosening, rods shedding and loosening. If these adverse events occur, the patient should be treated accordingly.

\section{Assessment of outcome}

The clinical outcome was measured by the visual analog scale(VAS) score ( $0=$ no pain, $10=$ worst imaginable pain) of axial neck pain (neck, neck or shoulder pains). The preoperative and postoperative neurological condition was evaluated by the Japanese Orthopaedic Association (JOA) score [16]. The excellent rate proposed by the method by Hirabayashi [17] was calculated using the following formula: Recovery rate $(\%)=[($ postoperative JOA score冈preoperative JOA score) /(17囚preoperative JOA score)]× 100. The criteria for efficacy grading are: excellent, recovery rate $\geq 75 \%$; good, $50 \%$ to $74 \%$; fair, $25 \%$ to $49 \%$; poor, $<25 \%$. The incidence of adverse reactions was calculated and used to evaluate the safety of the procedure. The incidence of adverse reactions $=$ the number of patients with adverse reactions $/$ the total number of patients $\times 100 \%$.

\section{Radiographic evaluation}

Outpatient follow-up was performed regularly at 3, 6, 12, 24, and 36 months after surgery. All patients underwent cervical spine radiographs at each follow-up (including lateral, neutral, flexion, and extension views). Cervical curvature index ( $\mathrm{CCl}$ ) and cervical range motion (ROM) were calculated. The $\mathrm{CCl}$ was calculated by measuring the angle between the upper edge of $\mathrm{C} 3$ and the lower edge of $\mathrm{C} 7$ on the lateral 
radiograph of the cervical spine. The ROM was calculated by measuring the difference of included angle between $\mathrm{C} 2$ and $\mathrm{C} 7$ vertebral lower margin on lateral X-ray films of cervical hyperextension view and hyperflexion view. CT scan was used to evaluate the degree of spinal canal enlargement and bony unions of the grooves. MRI was used to evaluate the change of signal intensity and the decompression condition of the spinal cord.

\section{Data management}

Data were collected in the form of a Case Report Form (CRF) and summarized in a unified form. Specific data include the following aspects: demographic information, disease diagnosis, disease concomitant conditions and adverse reactions. These data are recorded and saved as electronic files by using Epidata data processing software. Research data of this clinical trial are kept by the First Affiliated Hospital of Zhengzhou University. Only researchers have the right to inquiry the documents of the database. The Independent Data Monitoring Committee (IDMC) supervises and manages data throughout the clinical research process. Professional statisticians will make a statistical analysis of the database files and get the statistical analysis results. The main researchers of the trial then write the research report based on the statistical results.

\section{Statistical analysis}

All statistical analyses are performed with SPSS V.21.0 statistical software (SPSS, Inc, Chicago, IL). All data are expressed as the mean \pm standard deviation. The difference of incision length, operative time, and intraoperative blood loss between the two groups will be analyzed using a two independent samples t-tests $(a=0.05)$. JOA score, VAS score, CCI, ROM and the sagittal diameter of the spinal canal of preoperatively, 1 week postoperatively, as well as $3,6,12,24$ months postoperatively will be analyzed using a repeated-measures analysis of variance. A value of $P<0.05$ is considered statistically significant.

\section{Discussion}

3D printed guiding device assisted bilateral crossing cervical microendoscopic laminoplasty is a new minimally invasive spinal surgical technique. The innovations of this new surgical technique are as follows: (a) By using 3D printing technology, customized ends of the guiding tubes were perfectly matched with the surface of lamina for surgical locating, achieving the purpose of minimally invasive and precise surgery; (b) The guiding grooves can prevent long groove-shaped decompression of the lamina from deflecting; (c) The surgical operating device for the bilateral crossing cervical microendoscopic laminoplasty can achieve accurate decompression of the lamina, rapid fixation of lateral mass screws and precise retroposition of the spinous process-ligament complex; (d) Through preoperative simulation, the position of the decompression grooves and the entry point of the lateral mass screws were determined to prevent complications.

This procedure can effectively enlarge the spinal canal while retaining most of the normal bone-ligament anatomical structure of the cervical spine. After full decompression, two lateral mass screws were 
vertically twisted into the lateral mass on both sides. The connecting rod was twisted into the root of the spinous process through the aiming device and then fixed with the lateral mass screw. By adjusting the retroposition locking mechanism of the lateral mass screw to make the bilateral connecting rods retroposition 1 to $3 \mathrm{~mm}$ on the sagittal plane, thereby the spinal canal was enlarged. With the help of the bilateral connecting rods, the spinous process-ligament complex and the lateral mass screws formed a stable triangular structure in posterior column of this level which further reducing the risk of postoperative spinal instability.

Indications of 3D printed guiding device assisted bilateral crossing cervical microendoscopic laminoplasty are as follows: (a) Congenital spinal canal stenosis; (b) Spinal canal stenosis caused by ossification of the posterior longitudinal ligament, hypertrophy of ligamentum flavum, and proliferation of articular process; (c) The dura mater is compressed by multi-level cervical disc herniation; (d) The anterior cervical operation cannot alleviate symptoms. Contraindications include patients with cervical kyphosis, cervical instability, cervical spondylolisthesis over 1 grade and severe osteoporosis.

This trial was the first randomized controlled trial to compare 3D printed guiding device assisted bilateral crossing cervical microendoscopic laminoplasty with CMEL. The purpose of the trial was to introduce a new minimally invasive laminoplasty technique and to demonstrate that this new surgical method is safer, easier and more effective.

\section{Trial status}

This trial was registered on 11 May 2019 (registration number ChiCTR1900023100). The trial is currently in the stage of recruiting patients. The trial began recruitment in 1 June 2019. Participants will be recruited until 1 June 2020.

\section{Abbreviations}

CMEL: Cervical microendoscopic laminoplasty; CSM: Cervical spondylotic myelopathy; JOA: Japanese Orthopedic Association scores; VAS: Visual analogue scales; CCI: Cervical curvature index; ROM: Range of motion; RCT: Randomized controlled trial; ChiCTR: Chinese Clinical Trial Registry; WHO ICTRP: World Health Organization International Clinical Trials Registry Platform; MRI: Magnetic Resonance Imaging; CT: Computed Tomography; PASS: Power Analysis and Sample Size; CRF: Case Report Form; IDMC: Independent Data Monitoring Committee; SPSS: Statistical Product and Service Solutions; SPIRIT:

\section{Standard Protocol Items Recommendations for Intervention Trials Declarations}

\section{Acknowledgements}

The authors would especially like to thank engineer Peng Li and engineer Jun Li for their assistance in preparing the manuscript. 


\section{Funding}

This trial was conducted with no external funding and was instead funding from internal funding. Internal funding come from Department of Orthopaedics, the First Affiliated Hospital of Zhengzhou University, China.

\section{Availability of data and materials}

Not applicable.

\section{Authors' contributions}

All authors are affiliated to the First Affiliated Hospital of Zhengzhou University, China. CLZ designed the study protocol. YF wrote the manuscript. CLZ and YX checked the manuscript. CLZ revised and finalized the study protocol. $X Y, T Y Y, C L S, L J S, Y L, Y Y W$ and $X Z$ are responsible for the recruitment and treatment of patients. All authors have read and approved the final manuscript.

\section{Ethics approval and consent to participate}

This trial meets the requirements of the Helsinki Declaration formulated by the World Medical Association, and it has been reviewed and approved by the Institutional Ethics Review Board of the First Affiliated Hospital of Zhengzhou University (Approved number: KY-2019-LW-049). All enrolled participants will be required to sign an informed consent. The protocol has been registered in Chinese Clinical Trial Registry (ChiCTR), assigned to be the representative registry of China to join WHO ICTRP in 2007, with the protocol number ChiCTR1900023100.

\section{Consent for publication}

Not applicable.

\section{Competing interests}

The authors declare that they have no competing interests.

\section{Data sharing statement}

The full data set will be made available when this trial is completed and published.

\section{Publisher's Note}

Springer Nature remains neutral with regard to jurisdictional claims in published maps and institutional affiliations.

\section{Open Access}


This article is distributed under the terms of the Creative Commons Attribution 4.0 International License (http://creativecommons.org/licenses/by/4.0/), which permits unrestricted use, distribution, and reproduction in any medium, provided you give appropriate credit to the original author(s) and the source, provide a link to the Creative Commons license, and indicate if changes were made. The Creative Commons Public Domain Dedication waiver (http://creativecommons.org/publicdomain/zero/1.0/) applies to the data made available in this article, unless otherwise stated.

\section{References}

1. Melancia JL, Francisco AF, Antunes JL. Spinal stenosis. Handb Clin Neurol 2014;119:541-9.

2. Seichi A, Takeshita K, Ohishi I, et al. Long-term results of double-door laminoplasty for cervical stenotic myelopathy. Spine 2001;26:479-87.

3. Chiba K, Ogawa Y, Ishii K, et al. Long-term results of expansive open-door laminoplasty for cervical myelopathy-average 14-year follow-up study. Spine 2006;31:2998-3005.

4. Hosono N, Yonenobu K, Ono K. Neck and shoulder pain after laminoplasty. A noticeable complication. Spine 1996;21:1969-73.

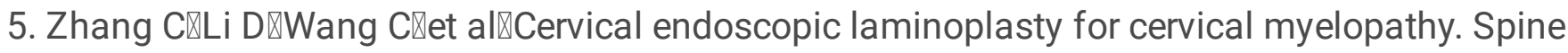
2016;41: B44-B51.

6. Adamson TE. Microendoscopic posterior cervical laminoforaminotomy for unilateral radiculopathy: results of a new technique in 100 cases. J Neurosurg 2001;95(1 Suppl):51-7.

7. Fessler RG, Khoo LT. Minimally invasive cervical microendoscopic foraminotomy: an initial clinical experience. Neurosurgery 2002;51:S37-45.

8. Coric D, Adamson T. Minimally invasive cervical microendoscopic laminoforaminotomy. Neurosurg Focus 2008;25:E2.

9. Yabuki S, Kikuchi S. Endoscopic partial laminectomy for cervical myelopathy. J Neurosurg Spine 2005;2:170-4.

10. Minamide A, Yoshida M, Yamada H, et al. Clinical outcomes of microendoscopic decompression surgery for cervical myelopathy. Eur Spine J 2010;19:487-93.

11. Dahdaleh NS, Wong AP, Smith ZA, et al. Microendoscopic decompression for cervical spondylotic myelopathy. Neurosurg Focus 2013;35:E8.

12. Yadav YR, Parihar V, Ratre S, et al. Endoscopic decompression of cervical spondylotic myelopathy using posterior approach. Neurol India 2014;62:640-5.

13. Oshima $\mathrm{Y}$, Takeshita $\mathrm{K}$, Inanami H, et al. Cervical microendoscopic interlaminar decompression through a midline approach in patients with cervical myelopathy: a technical note. J Neurol Surg A Cent Eur Neurosurg 2014;75:474-8.

14. Chan AW, Tetzlaff JM, Gøtzsche PC, et al. SPIRIT 2013 explanation and elaboration: guidance for protocols of clinical trials. BMJ 2013;346:e7586. 
15. Jeanneret B, Magerl F, Ward EH, et al. Posterior stabilization of the cervical spine with hook plates. Spine (Phila Pa 1976) 1991;16:S56-63.

16. Yonenobu K, Abumi K, Nagata K, et al. Interobserver and intraobserver reliability of the japanese orthopaedic association scoring system for evaluation of cervical compression myelopathy. Spine(Phila Pa 1976) 2001;26:1890-5.

17. Hirabayashi K, Toyama Y. Choice of surgical procedure for cervical ossification of the posterior Iongitudinal ligaments. In:Yonenobu K, Sakou T, Ono K, editors. Ossification of the Posterior Longitudinal Ligament. Tokyo: Springer 1997.pp. 135-142.

\section{Figures}




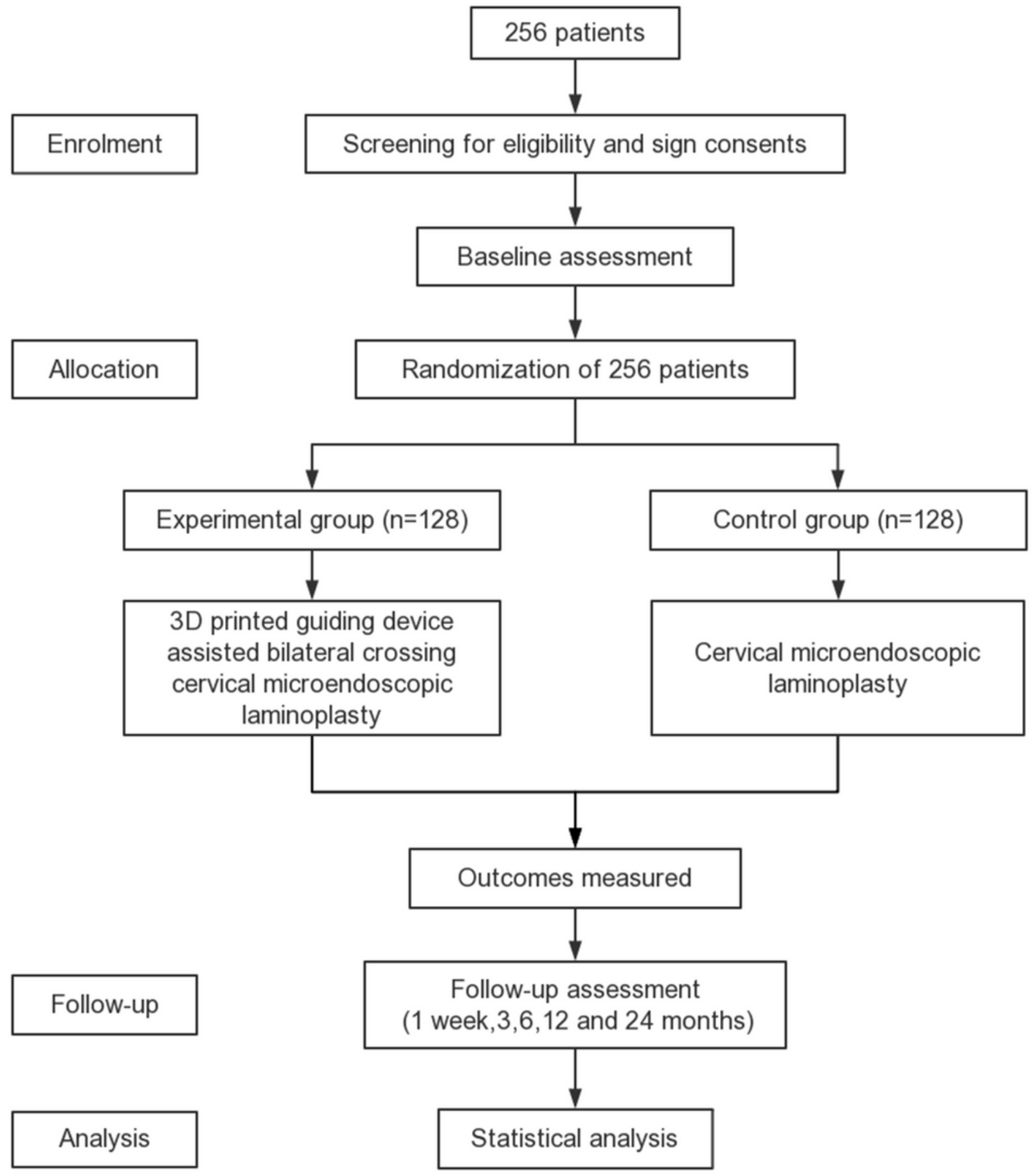

\section{Figure 1}

flow chart of the trial. 


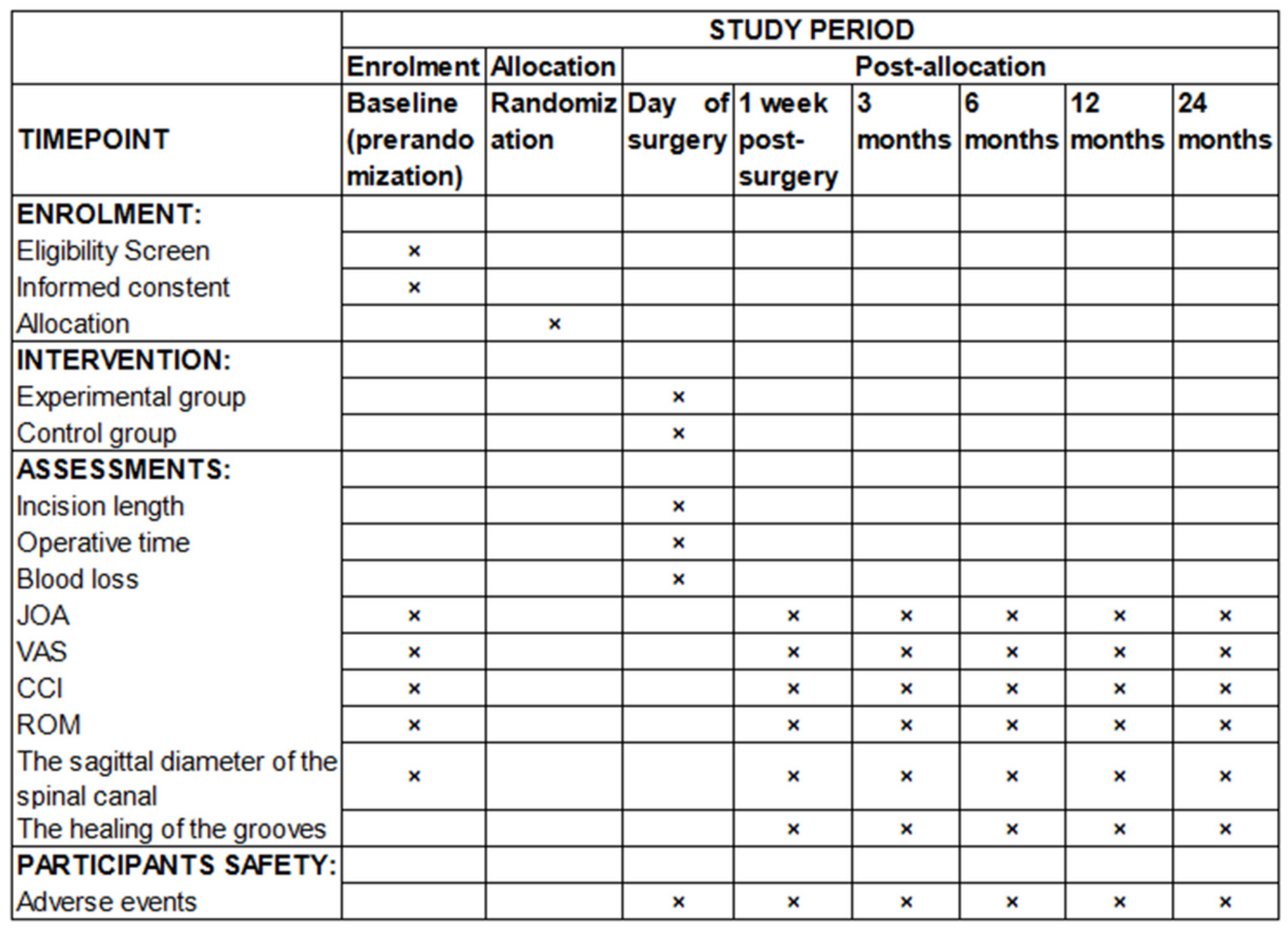

Figure 2

Standard Protocol Items: Recommendations for Interventional Trials (SPIRIT) schedule of the trial. 


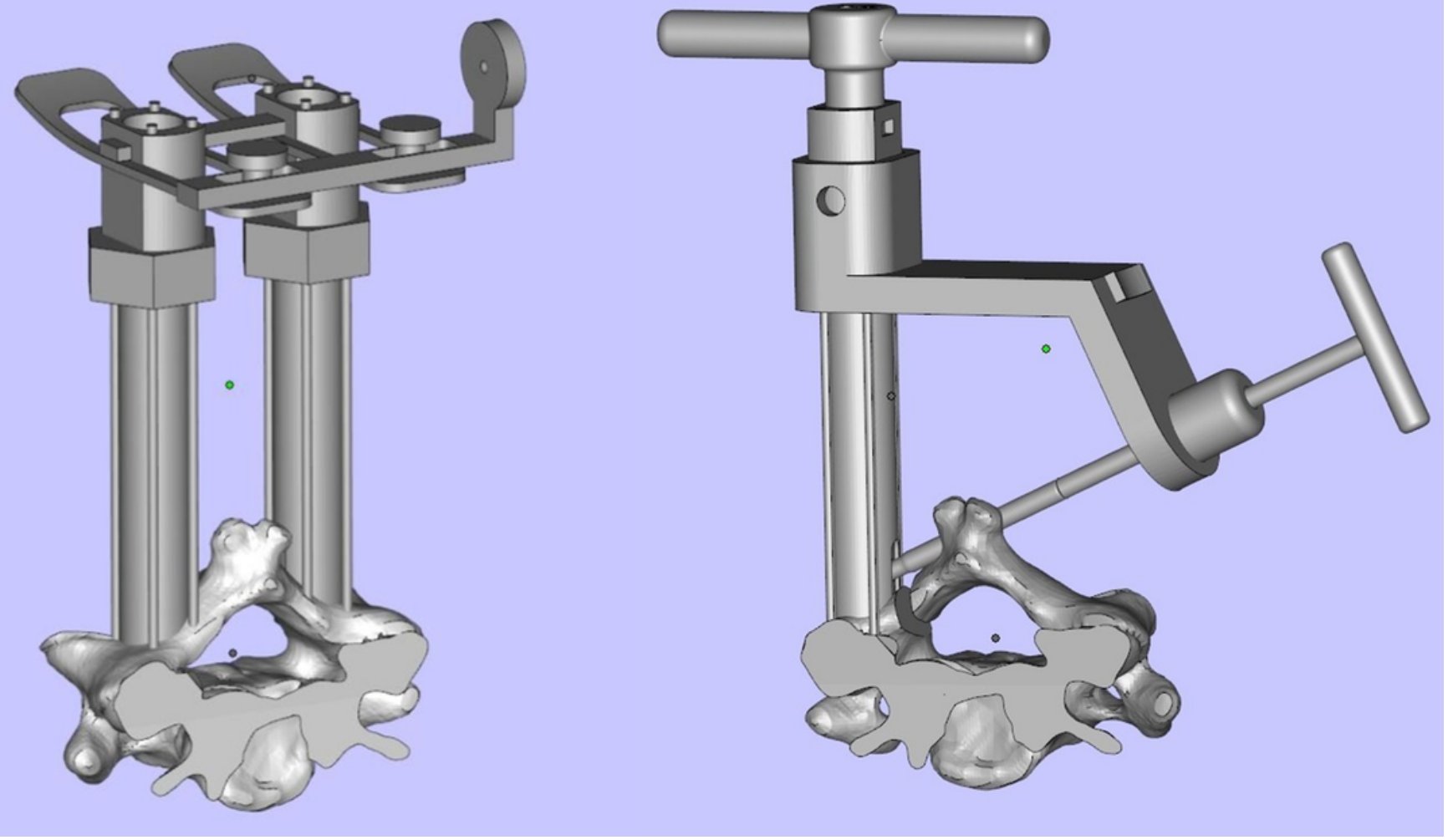

\section{Figure 3}

The designed guiding tubes, guiding grooves and aiming device in E3D Medical Software. 


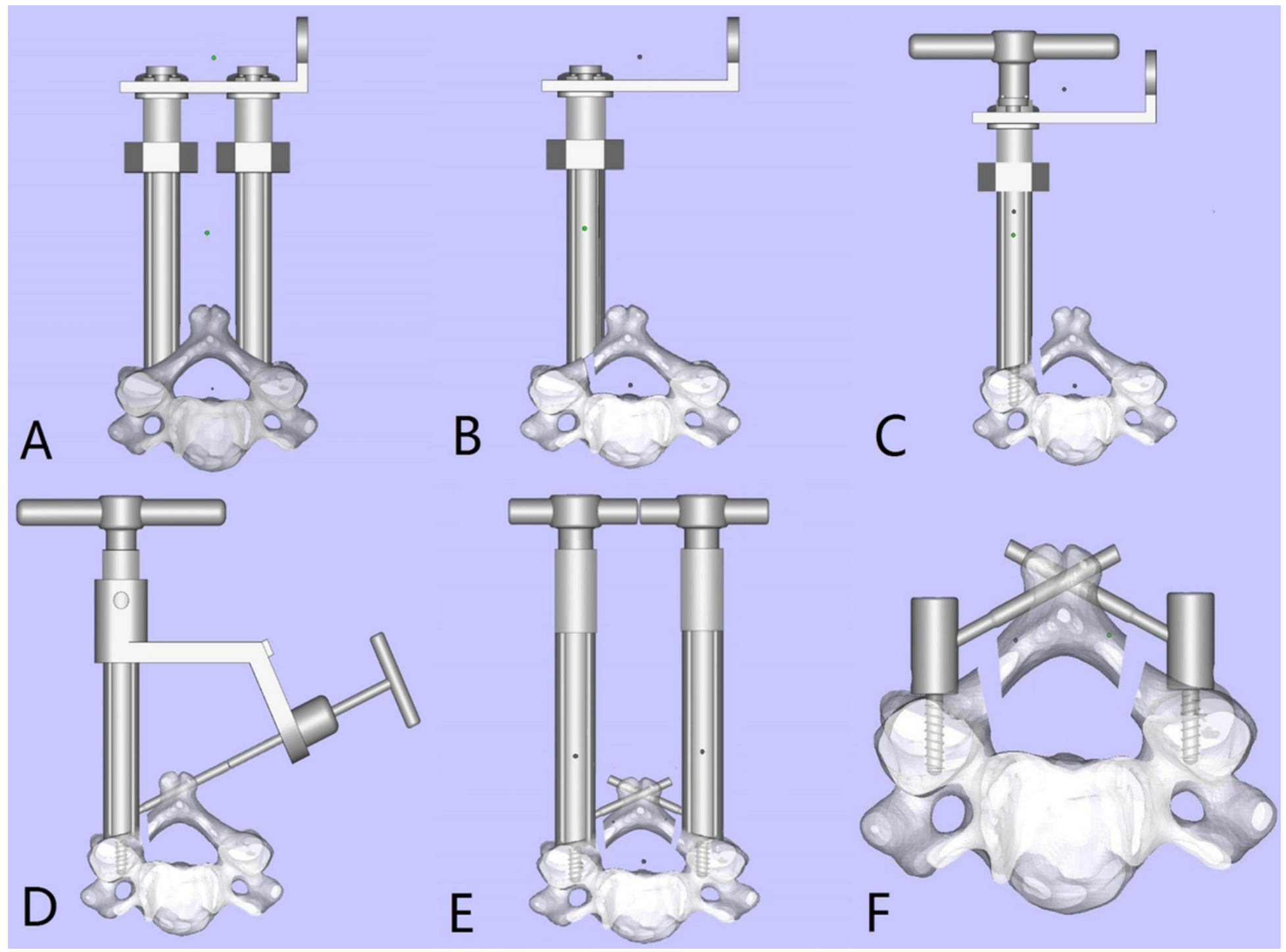

\section{Figure 4}

Diagrammatic sketch of the 3D printed guiding device assisted bilateral crossing cervical microendoscopic laminoplasty. (A) The two guiding tubes were connected with the bilateral guiding grooves. After the two guiding tubes passed through the incisions on both sides, customized ends of the guiding tubes were perfectly matched with the surface of lamina by adjusting the guiding tube, and confirmed by the indicators. (B) The guiding tube on the one side was removed. Then the endoscope was inserted into the guiding tube on the contralateral side. Endoscopic partial laminotomy was initiated using a high-speed drill to roughen the conjunction of the lamina and the lateral mass, and the ventral cortex of the lamina was completely removed by using a $2 \mathrm{~mm}$ Kerrison laminectomy rongeur. (C) The guiding tube was removed towards the lateral side by a certain distance, then the lateral mass screw was fixed at the lateral mass with the help of the working sleeve. (D) The aiming device was installed on the guiding tube, then the connecting rod was passed through the root of the spinous process by using the aiming device, and it was fixed at the lateral mass screw. (E) The same process was repeated on the contralateral side. Then two working sleeves were connected with the bilateral lateral mass screws. By adjusting the retroposition locking mechanism of the lateral mass screw to make the bilateral connecting 
rods retroposition 1 to $3 \mathrm{~mm}$ on the sagittal plane, therefore, the spinous process-ligament complex and the deep extensor muscle group also retroposition 1 to $3 \mathrm{~mm}$ on the sagittal plane. $(F)$ With the help of the bilateral connecting rods, the spinous process-ligament complex and the lateral mass screws formed a stable triangular structure in posterior column of this level.

\section{Supplementary Files}

This is a list of supplementary files associated with this preprint. Click to download.

- SPIRIT.pdf 